\title{
Time-domain full waveform inversion using the gradient preconditioning based on seismic wave energy: Application to the South China Sea
}

\author{
Zhong Mengxuan ${ }^{1}$, Tan Jun*l, Song Peng ${ }^{1}$, Zhang Xiao-bo ${ }^{l}$, Xie Chuang ${ }^{1}$, Liu Zhao-lun ${ }^{2}$ \\ ${ }^{1}$ Ocean University of China \& Qingdao National Laboratory for Marine Science and Technology \& Key Lab of \\ Submarine Geosciences and Prospecting Techniques Ministry of Education, ${ }^{2}$ King Abdullah University of \\ Science and Technology
}

\section{Summary}

The gradient preconditioning algorithms based on Hessian matrices in time-domain full waveform inversion (FWI) are widely used now, but consume a lot of memory and do not fit the FWI of large models or actual seismic data well. To avoid the huge storage consumption, the gradient preconditioning approach based on seismic wave energy has been proposed it simulates the "approximated wave field" with the acoustic wave equation and uses the energy of the simulated wavefield to precondition the gradient. The method does not require computing and storing the Hessian matrix or its inverse and can effectively eliminate the effect caused by geometric diffusion and uneven illumination on gradient. The result of experiments in this article with field data from South China Sea confirms that the time-domain FWI using the gradient preconditioning based on seismic wave energy (GPWE) can achieve higher inversion accuracy for the deep high-velocity model and its underlying strata.

\section{Introduction}

As the gradient of traditional FWI is affected by geometric diffusion and uneven illumination, the velocity updates in the middle-shallow strata always dominate the inversion process. Accordingly, the inversions in the deep strata and even the whole region have slower convergence and accuracy. Many solutions have been proposed to address this problem, including the depth-weighted gradient method, the layer-by-layer inversion (Wang, 2009), and the gradient preconditioning based on Hessian matrices (Hessian matrix, approximated Hessian matrix, pseudoHessian matrix, and limited-memory Broyden-FletcherGoldfarb-Shanno (L-BFGS) algorithm). Relying on depth weighting formula, the depth-weighted method is unsuitable for the inversion of complex models. Besides, it is also difficult to achieve high-accuracy inversion by layer-by-layer method due to the deficiency of objective criteria for horizontal division and the difficulty in intercepting shot-gather time window. The preconditioning algorithms based on Hessian matrices are widely used now. However, the algorithms based on Hessian matrix and the approximated Hessian matrix (Pratt, 1998; Shin, 2001), usually consume a lot of memory, and are not suitable for the FWI of large models or actual seismic data. The algorithm based on the pseudo-Hessian matrix (Choi et al,
2008) fails to achieve an accurate inversion in the deep strata. As for the L-BFGS algorithm (Brossier et al, 2009; DiaZ et al, 2011; Zhang et al, 2013), a certain number of gradient and variable residuals need to be stored (Wang, 2013). The convergence of ill-posed problems is slow due to its limited capacity in collecting curvature information (Morales and Nocedal, 2002; Wang, 2013).

A gradient preconditioning method based on seismic wave energy has been proposed by Zhang et al $(2011,2012 \mathrm{a}$, 2012 b), in order to avoid computing and storing large-scale matrix and eliminate the impact caused by geometric diffusion and uneven illumination on gradient. The method does not require computing and storing the Hessian matrix and its inverse. Meanwhile, it considerably raises the inversion accuracy in the deep strata. Based on this method, we perform experiments on field data to illustrate its inversion result.

\section{Theory}

Tarantola et al $(1984,1986)$ derived the gradient that can be directly applied to the time-domain FWI from the Born approximation. When density is a constant, the gradient calculation equation can be written as

$$
\gamma(\boldsymbol{x})=\frac{2}{c(\boldsymbol{x})^{3}} \sum_{s, r} \int \dot{\psi}\left(\boldsymbol{x}, t, \boldsymbol{x}_{\boldsymbol{r}}\right) \dot{p}\left(\boldsymbol{x}, t, \boldsymbol{x}_{s}\right) d t
$$

where $\boldsymbol{x}$ denotes the spatial position of the model; $c(x)$ is the velocity at point $\boldsymbol{x} ; p\left(\boldsymbol{x}, t, \boldsymbol{x}_{s}\right)$ stands for the forward time wavefield with the source wavelet at $\boldsymbol{x}_{s} ;\left(\boldsymbol{x}, t, \boldsymbol{x}_{r}\right)$ represents the reverse time wavefield with the virtual source at $\boldsymbol{x}_{r}$; the superscript "." signifies the first-order differential of time.

The gradient calculated by Eq. (1) is influenced by geometric diffusion and uneven illumination in forward time and reverse time wavefields. Without preconditioning, the velocity updates in the middle-shallow strata will dominate the inversion process, thereby resulting in slower convergence and lower inversion accuracy in the deep strata and even the whole region. Pratt et al (1998) indicated that the Hessian matrix can precisely reflect the degree of geometric diffusion and illumination unevenness of wavefields. Therefore, the influence of geometric diffusion and uneven illumination on gradient can be effectively eliminated by preconditioning the Hessian matrix (i.e., the gradient calculated by Eq. (1) is multiplied by the inverse matrix of the Hessian matrix to produce the 


\section{Time-domain FWI using the GPWE: Application to the South China Sea}

final gradient for inversion). In this way, the convergence efficiency and accuracy of the FWI can be promoted significantly.

The Hessian matrix $\mathbf{H}$ is the second-order partial derivative of the objective function with respect to the model $\mathrm{m}$. It can be written as

$$
\mathbf{H}=\frac{\partial^{2} E}{\partial \mathbf{m}^{2}}=\frac{\partial \boldsymbol{\gamma}}{\partial \mathbf{m}}
$$

The formula can be further arranged as

$$
\mathbf{H}=\mathbf{A}^{T} \mathbf{A}+\left(\frac{\partial \mathbf{A}}{\partial \mathbf{m}}\right)^{T} \delta \mathbf{d}
$$

The first term in the Hessian matrix plays a leading role, so the second term is ignored, leading to the approximated (Guass-Newton) Hessian matrix:

$$
\mathbf{H}_{\boldsymbol{\alpha}}=\mathbf{A}^{T} \mathbf{A}
$$

The diagonal elements on the approximated Hessian matrix act as the primary regulator of gradient (Pratt, 1998). Therefore, these diagonal elements or their approximations are generally applied in actual inversion to precondition the gradient (Shin, 2001; Choi, 2008). However, such preconditioning usually requires huge computing and storing effort.

When the density of underground medium is a constant, Jacobian matrix can be written as (Zhang, 2012a)

$$
\mathbf{A}(\boldsymbol{x})=\sum_{(s, r)} \int \dot{g}\left(\boldsymbol{x}, t, \boldsymbol{x}_{\boldsymbol{r}}\right) \dot{p}\left(\boldsymbol{x}, t, \boldsymbol{x}_{s}\right) d t
$$

where $g$ is Green's function; $g\left(\boldsymbol{x}, t, \boldsymbol{x}_{r}\right)$ signifies the reverse time wavefield value at $\boldsymbol{x}$ resulting from the impulse disturbance at $\boldsymbol{x}_{r} ; p\left(\boldsymbol{x}, t, \boldsymbol{x}_{s}\right)$ stands for the forward propagating wavefield value at $x$ resulting from the source disturbance at $\boldsymbol{x}_{s}$; the superscript "." denotes the first-order time derivative. Denoting the discrete time series of $\dot{p}\left(\boldsymbol{x}, t, \boldsymbol{x}_{\boldsymbol{s}}\right)$ as $U\left(\boldsymbol{x}, t, \boldsymbol{x}_{s}\right)$ and $\dot{g}\left(\boldsymbol{x}, t, \boldsymbol{x}_{\boldsymbol{r}}\right)$ as $G\left(\boldsymbol{x}, t, \boldsymbol{x}_{s}\right)$, the diagonal elements of the approximated Hessian matrix can be written as

$$
\begin{aligned}
\operatorname{diag}\left\{\mathbf{A}^{T} \mathbf{A}\right\}= & \left\{\sum_{(s, r, t)}\left[U\left(\boldsymbol{x}_{1}, t, \boldsymbol{x}_{s}\right) G\left(\boldsymbol{x}_{1}, t, \boldsymbol{x}_{r}\right)\right]^{2},\right. \\
& \left.\cdots, \sum_{(s, r, t)}\left[U\left(\boldsymbol{x}_{M}, t, \boldsymbol{x}_{s}\right) G\left(\boldsymbol{x}_{M}, t, \boldsymbol{x}_{r}\right)\right]^{2}\right\}
\end{aligned}
$$

where $\boldsymbol{x}_{i}(i=1,2, \ldots, M)$ denotes the specific spatial position. We can know from Eq. (6) that the regulation of the approximated Hessian matrix on the gradient is essentially a preconditioning on gradient with energy values of forward time and reverse time wavefields. As a result, to avoid direct computation and storage of the approximate Hessian matrix, Zhang et al (2011, 2012a, 2012b) put forward a gradient preconditioning method based on seismic wave energy. In their studies, the energy values of forward time and reverse time wavefields are defined as

$$
\begin{aligned}
& W_{s}(\boldsymbol{x})=\sum_{(s, t)} p^{2}\left(\boldsymbol{x}, t, \boldsymbol{x}_{s}\right) \\
& W_{r}(\boldsymbol{x})=\sum_{(s, r, t)} \phi^{2}\left(\boldsymbol{x}, t, \boldsymbol{x}_{\boldsymbol{r}}\right)
\end{aligned}
$$

where $p\left(\boldsymbol{x}, t, \boldsymbol{x}_{s}\right)$ denotes the forward time wavefield from the seismic source, which takes the source wavelet as a disturbance and can be obtained from the forward modelling of the acoustic wave equation; $\phi\left(x, t, \boldsymbol{x}_{r}\right)$ indicates the reverse time wavefield at the receiver point, which takes the simulated record $u\left(\boldsymbol{x}_{r}, \boldsymbol{x}_{s}\right)$ as a disturbance and can be obtained from the reverse time extrapolation of the acoustic wave equation. $\gamma_{p}$ is the gradient after preconditioning and defined (Zhang, 2012a) as follow:

$$
\gamma_{P}(\boldsymbol{x})=\frac{\gamma(\boldsymbol{x})}{\sqrt{W_{s}(\boldsymbol{x}) W_{r}(\boldsymbol{x})}}
$$

In essence, the gradient preconditioning method based on seismic wave energy is to describe the degree of geometric diffusion and illumination unevenness with the energy of seismic wave and further precondition the gradient. It avoids computing and storing high-dimensional matrices in those algorithms based on Hessian matrices and effectively overcome the influence of geometric diffusion and uneven illumination on gradient. Zhang et al (2012b) has confirmed in his experiment that compared with the preconditioning based on the pseudo-Hessian matrix, higher inversion accuracy can be obtained when using the gradient preconditioning based on seismic wave energy.

\section{Examples}

A field survey line in the South China Sea was selected for FWI, where 1,037 shots were arranged on the right side and receivers on the left side. Each shot had 240 receivers. Both shots and receivers had a 25-meter wide interval and the minimum offset was $100 \mathrm{~m}$. Both shot and receiver were $20 \mathrm{~m}$ deep and the record length of the shot gather was $3.5 \mathrm{~s}$ (the record of the 650th shot is given in Figure 1).

The $15.5 \mathrm{~km}$ to $32 \mathrm{~km}$ section was cut out from the survey line for inversion test. Firstly, a seismic wavelet was extracted from the original shot gather data as the source of the inversion. A velocity model for the pre-stack depth migration was established with relevant analytical technique as the initial velocity model in the inversion (seismic wavelet and initial velocity model are shown in Figure 2 and Figure 3, respectively). Then the FWI using the gradient preconditioning based on seismic wave energy was performed at three high cutoff frequencies $(15 \mathrm{~Hz}$, $27 \mathrm{~Hz}, 55 \mathrm{~Hz}$ ), with 100 iterations for each. See Figure 4 for final inversion results. 


\section{Time-domain FWI using the GPWE: Application to the South China Sea}
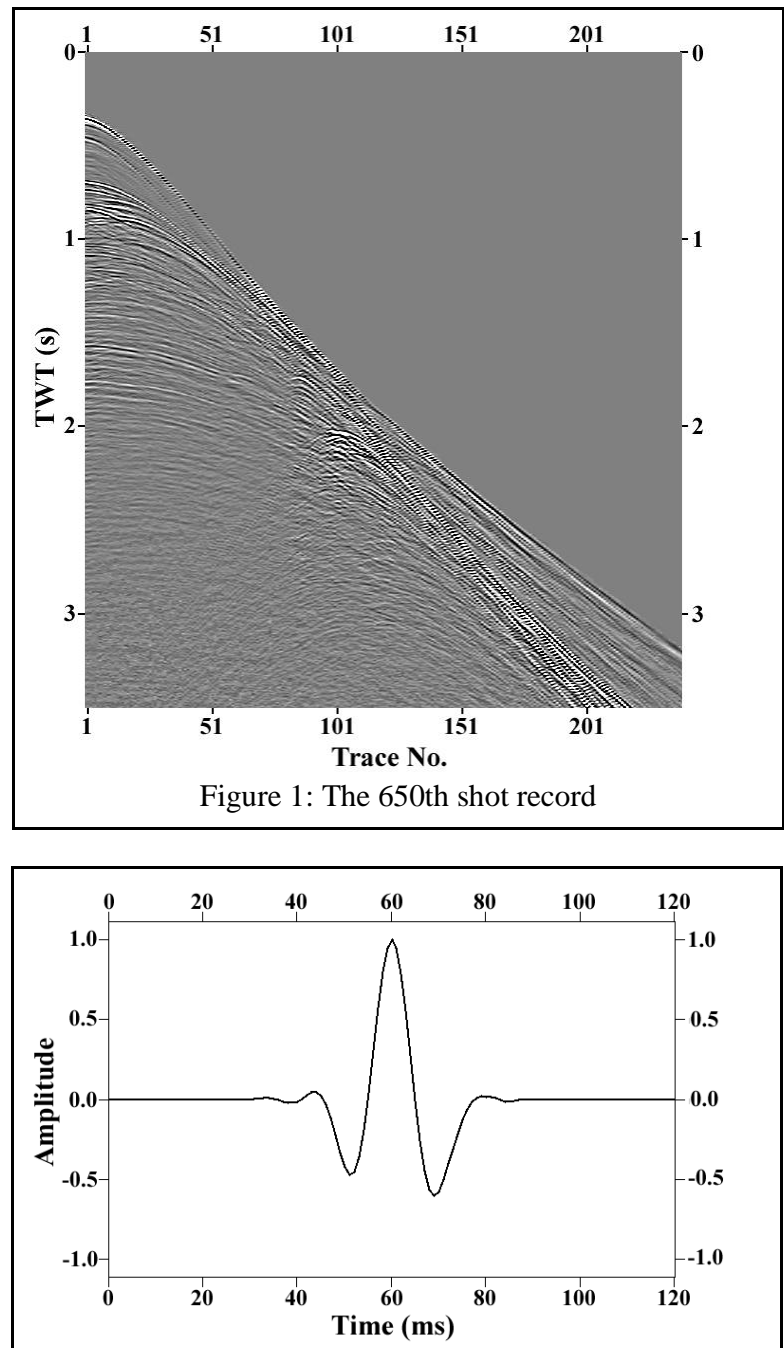

Figure 2: Seismic wavelet

Reverse-time migration was carried out based on the final inversion results, and the section of reverse-time migration is shown in Figure 5. Comparing Figure 4 and Figure 5, it can be found that the final inversion results were in high accordance with tectonic characteristics of the migration section, indicating that the method we proposed is highly applicable to the FWI in field data.

\section{Conclusion}

We put forward the gradient preconditioning approach based on the energy of seismic waves. It preconditions the gradient using the seismic energy simulated by the acoustic wave equation. The approach avoids computing and storing high-dimensional matrices in those algorithms based on Hessian matrices and effectively eliminates the influence of geometric diffusion and uneven illumination on gradient. An application to the South China Sea shows that the timedomain FWI using the gradient preconditioning approach performs well even for complex models, which validates the applicability of the proposed method to the FWI in field data. Moreover, compared with the inversion using the gradient preconditioning based on seismic wave energy, this method can achieve higher accuracy for deep highvelocity models and their underlying strata.

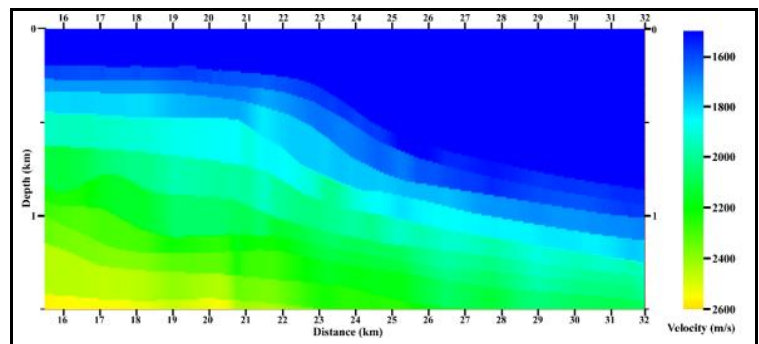

Figure 3: The initial velocity model

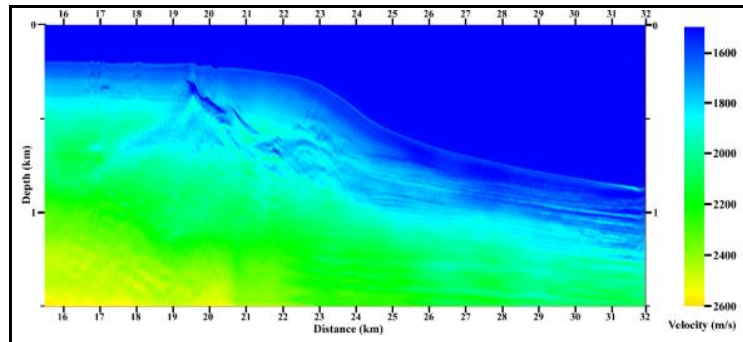

Figure 4: The inversion result

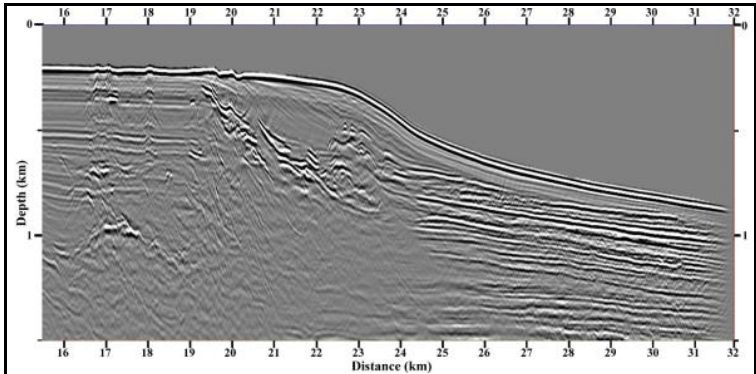

Figure 5: The section of reverse-time migration

\section{References}

Brossier R, Operto S, and Virieux J. 2009. Seismic Imaging of complex onshore structures by 2D elastic frequencydomain full-waveform inversion. Geophysics, 74(6): WCC105- WCC118. 


\section{Time-domain FWI using the GPWE: Application to the South China Sea}

Choi Y, Min D J, Shin C. 2008. Frequency-domain elastic full waveform inversion using the new pseudo-Hessian matrix: Experience of elastic Marmousi-2 synthetic data. Bulletin of the Seismological Society of America, 98(5): 2402-2415.

Diaz Esteban. 2011. Fast full waveform inversion with random shot decimation. 81th Annual International Meeting, SEG, Expanded Abstracts, 2804-2808.

Morales, J L, and Nocedal, J. 2002. Enriched methods for large-scale unconstrained optimization. Computational Optimization and Applications, 143-154.

Pratt G, Shin C. 1998. Gauss-Newton and full Newton methods in frequency-space seismic waveform inversion. Geophysical Journal International, 133(2): 341-362.

Shin C, Yoon K G, Marfurt K J, et al. 2001. Efficiemt calculation of a partial-derivative wavefield using reciprocity for seismic imaging and inversion. Geophysics, 66(6):1856-1863.

Tarantola A. 1984. Inversion of seismic reflection data in the acoustic approximation. Geophysics, 49(8): 1259-1266.

Tarantola A. 1986. A strategy for nonlinear elastic inversion of seismic reflection data. Geophysics, 51(10): 1893-1903.

Wang Y, Dong Liang-guo, and Liu Yu-zhu. 2013. Improved hybrid iterative optimization method for seismic full waveform inversion. Applied Geophysics, 10(3): 265277.

Wang Y, Rao Y. 2009. Reflection seismic waveform tomography, Journal of Geophysical research, 114(B3): B03304.

Zhang Zhi-gang, Lin You-zuo and Huang Lian-jie. 2011. Full-waveform inversion in the time domain with an energy-weighted gradient. 81th Annual International Meeting, SEG, Expanded Abstracts, 2772-2776.

Zhang Zhi-gang, Huang Lian-jie and Lin You-zuo. 2012a. A wave-energy-based precondition approach to fullwaveform inversion in the time domain. 82th Annual International Meeting, SEG, Expanded Abstracts, 1-5.

Zhang Zhi-gang, Huang Lian-jie and Lin You-zuo. 2012b. Double-difference elastic-waveform inversion with weighted gradients for monitoring EGS reservoirs. 37th Workshop on Geothermal Reservoir Engineering Stanford University, SGP-TR-194.
Zhang Sheng-qiang, Liu Chun-cheng, Han Li-guo, Yang Xiao-chun. 2013. Frequency multi-scale full waveform inversion based on L-BFGS algorithm simultaneous sources approach. Journal of Jilin University: Earth Science Edition (in Chinese), 43(3): 1004-1012. 